Recepción: 10 / 05 / 2018

Aceptación: 25 / 08 / 2018

Publicación: 05 / 10 / 2018

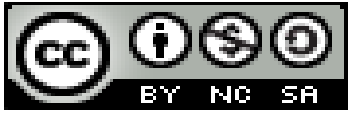

Ciencias de la educación

Artículo de investigación

\title{
Influencia de los juegos de concentración en el desarrollo de las relaciones lógico - matemáticas
}
Influence of concentration games on the development of logical-mathematical relations

\section{Influência de jogos de concentração no desenvolvimento de relaçães lógico- matemáticas}

\author{
Leonor Reyes-Gallo ${ }^{\mathrm{I}}$ \\ leonor.reyes@hotmail.com \\ Teresa Vargas-Cevallos II \\ heidy151@ hotmail.com \\ Lenin Mendieta-Toledo ${ }^{\text {III }}$ \\ lenin.mendietat@ug.eduec
}

Correspondencia: leonor.reyes@hotmail.com

\footnotetext{
I Magister en Docencia y Gerencia en Educación Superior, Licenciada en Ciencias de la Educación Especialidad Comercio y Administración, Profesora de Segunda Enseñanza Especialización Comercio y Administración, Docente Universidad de Guayaquil, Guayaquil, Ecuador.

${ }^{\text {II }}$ Docente Instituto Ana Paredes Alfaro, Guayaquil, Ecuador.

III Magister en Docencia Universitaria e Investigación Educativa, Master Universitario en Actividad Física y Salud, Licenciado en Ciencias de la Educación en la Especialidad de Educación Física, Docente Universidad de Guayaquil, Guayaquil, Ecuador.
} 


\section{Resumen}

El objetivo fue determinar la influencia de la aplicación de una guía didáctica basado en juegos de concentración sobre las relaciones lógico-matemáticos en estudiantes de 4 a 5 años de la Escuela de Educación Básica Mixta, "Eloy Alfaro”, del cantón Durán-Ecuador. La muestra la constituyeron 44 niños, fue una investigación correlacional, se aplicó la observación, encuesta y entrevista, además de un test pedagógico. Los resultados fueron un incremento del progreso adecuado, en un 50\% en participar en juegos grupales siguiendo las reglas y asumiendo roles que le permitan mantener un ambiente armónico con sus pares; un $47.6 \%$ en proponer juegos construyendo sus propias reglas interactuando con otros; un $43.4 \%$ en continuar y reproducir patrones simples con objetos concretos y representaciones gráficas; y, un incremento del 52,5\% en clasificar objetos con los atributos (tamaño, color o forma). Se disminuye los porcentajes de en proceso e iniciado. Se concluyó que la aplicación de un programa didáctico de juegos de concentración influye en el desarrollo de las relaciones lógico-matemáticas en los niños.

Palabras clave: juegos de concentración; relaciones lógico-matemáticas; programa didáctico.

\section{Abstract}

The aim of this research was to determine the influence of applying a didactic guide based on concentration games about logic-mathematical relations in students of 4 to 5 years old in the coeducational school for basic education, "Eloy Alfaro", from the city Durán in Ecuador. The set sample was 44 children, it was a correlational research, it was applied observation, survey, and interview, besides a pedagogical test. The results were an increase of the adequate progress, in $50 \%$ at group games participation following the rules and playing roles that allow them to be in a harmonic environment with their peers, $47.6 \%$ in proposing games building their own rules interacting with others; $43.4 \%$ in keeping and reproducing simple patterns with concrete objects and graphic representations; and increase of $52.5 \%$ in classifying objects with attributes (size, color, and shape). The percentages of in process and started decrease. The conclusion was that the application of a didactic program of concentration games affects the development of the logic-mathematical relations in children.

Key words: concentration games; logic-mathematical relations; didactic program. 


\section{Resumo}

O objetivo foi determinar a influência da aplicação de um guia didático baseado em jogos de concentração nas relações lógico-matemáticas em escolares de 4 a 5 anos da Escola de Educação Básica Mista, "Eloy Alfaro", do cantão Duran-Equador. A amostra foi composta por 44 crianças, foi uma investigação correlacional, a observação, levantamento e entrevista foram aplicados, bem como um teste pedagógico. Os resultados foram um aumento do progresso adequado, em $50 \%$ na participação em jogos de grupo seguindo as regras e assumindo papéis que lhe permitem manter um ambiente harmonioso com seus pares; 47,6\% em propor jogos construindo suas próprias regras interagindo com os outros; $43,4 \%$ para continuar e reproduzir padrões simples com objetos concretos e representações gráficas; e, um aumento de 52,5\% na classificação de objetos com os atributos (tamanho, cor ou forma). Diminui as porcentagens de em processo e iniciadas. Concluiu-se que a aplicação de um programa didático de jogos de concentração influencia o desenvolvimento de relações lógico-matemáticas em crianças.

Palavras chave: jogos de concentração; relações lógico-matemáticas; programa educacional.

\section{Introducción}

Delgado Linares \&, Inmaculada, (2011), definen al juego como "una actividad propia del ser humano, conducta característica de todo los tiempos, edades y culturas. Para el ser humano el juego adquiere una importancia clave en su desarrollo, especialmente en lo que refiere a su esfera global" (pág. 2). El juego de manera general con lleva un amplio conocimiento y dificultad a la hora de implementarse por eso, Esteban J. J., (2012) señala que el juego para el niño y la niña es "una forma innata de explorar el mundo, de conectarse con experiencias sensoriales, objetos, personas, sentimientos. Son en sí mismos ejercicios creativos de solución de problemas" (pág. 12). El juego es el medio más primitivo y especial de aprender de interrelacionarse con la comunidad que le rodea al ser humano, en este caso el desarrollo del niño en el transcurso de toda su vida, y así ir aprendiendo y relacionando todo lo que ocurre día a día de diferentes maneras y diversas formas con un solo propósito que es el avance personal. 
El juego de concentración es a criterio de Delgado Linares \& Inmaculada, (2011) quienes favorecen el desarrollo del pensamiento e insisten "El niño aprende que ciertos objetos encajan en otros, cuando se caen suenan, que una puede ponerse sobre otra sin que se caigan, el niño aprende a diferenciar formas, colores y texturas" (pág. 25). Como una actividad placentera, divertida, libre, entretenida y espontánea, sin propósito alguno, pero de gran uso y utilidad para el desarrollo del niño, desde la metacognición intrínseca que se produce al desarrollar habilidades y destrezas mientras aprende del objeto del conocimiento, esto con el pertinente acompañamiento del docente.

El conocimiento se fundamenta desde los primeros pasos de la escuela por eso es importante sus bases. Así Lev Vygotski, ya en 1931, indicaba al juego como el motor del desarrollo, Venegas, García, Venegas Rubiales, (2013) citan a Vygotsky quien manifiesta que un juego es "como el eje motor que permiten el afianzamiento de las zonas de desarrollo próximo, constituyéndose en parte de los deseos insatisfechos resueltos en situaciones simbólicas" (pág. 5). El arjé del proceso evolutivo del hombre, se produce debido a su innata curiosidad, a partir de lo cual surgen los juegos que a su vez ayudaron a aumentar la interacción del objeto del conocimiento con el sujeto cognoscente, el niño de esta manera crea, soluciona diferentes perspectivas para resolver problemas.

Los juegos son considerados la mejor estrategia en el aprendizaje de los niños, es por ellos que muchas investigaciones se centran en la lúdica como enfoque pedagógico para conseguir el empoderamiento del objeto de parte del sujeto cognoscente. A nivel de investigaciones de grado en el nivel superior se han realizado un sinnúmero de investigaciones al respecto Prasca medina, Pedroza Villarreal, \& Peña Zuñiga, (2016) permiten entender que las técnicas lúdicas son un medio eficaz de ayuda en el desarrollo del pensamiento, además de lograr desarrollar otras particularidades en el infante, de igual forma, las técnicas ludo-pedagógicas permiten al niño entretenerse mientras aprende de forma dinámica y didáctica, concomitantemente, logra despertar la curiosidad para crear y solucionar diferentes situaciones del educando, también sirve como un complemento en las relaciones docente- alumno, constituyéndose en el eje del aprendizaje en los niños. 
Cabanilla Guillén, (2016) permite desvelar la importancia de la lúdica desde el método activo de la lógica-matemática, la misma que ayuda al docente a implementar alternativas pedagógicas en el aula; Fonseca Ortega E., (2013) estableció la relación entre las actividades lúdicas, y el desarrollo del pensamiento lógico matemático y demostró que la utilización de este tipo de técnicas, ayudan al desarrollo del pensamiento lógico - matemático, motivan y despiertan el interés y la participación del estudiantado, quienes logran adquirir aprendizajes significativos desde la metacognición.

Es importante destacar, las apreciaciones de Huizinga, (1954) y Caillois, (1967), citados por Llull (2016) quines dan a conocer la importancia del juego como trasmisor cultural del desarrollo; además, los juegos practicados por alguna grey suelen determinar las características particulares de la misma, el juego es la alegría de los seres humanos y se convierten en entes gregarios cuando un nuevo juego aparece y se convierte en generador de esas sensaciones. Siempre la compañía en cualquier actividad que realizan las personas se ha dado cuenta que es más entretenida porque es especial ayuda a la interacción y complementación de un número más grande de personas en este caso los niños tienen a otros niños para poder relacionar y ver las diferentes maneras en que entre ellos realizan los juegos.

El juego como un acto de educar y socializar, Ibarra Rivas, (2017) permite inducir que el ser humano llega sin cultura que le permita ser persona humana, aunque esta humanización se da desde el vientre materno, continua con una secuencia de juego, educación, socialización, sin estos tres componentes el ser humano no es persona humana y este proceso perdura y se construye durante toda la vida, el juego a través de la educación que haga el trasvase de las normas, reglas y más condiciones, es a priori quien logre la socialización de la masa en su contexto, (pág. 14).

El conocimiento se lo adquiere mediante esfuerzo, aplicación y practica Según Brunner, indica la inducción al desarrollo, citando a Brunner; Giménez, (2014) señala "se refiere a la acción que puede desarrollar el adulto para llevar al niño a su nivel actual de conocimiento, a uno potencialmente más elevado" (pág. 22). Poder implementar el conocimiento en los niños requiere de un sinnúmero de destrezas que esto solo lo puede hacer la persona que está capacitado para hacerlo en este caso es el docente, es la persona que va a ayudar a construir el conocimiento 
desde cero y poder encaminarlo en la dirección correcta lo cual implica una relación mutua de los educados con el educador.

Carchi, (2012) indica "El conocimiento lógico-matemático es el que construye el niño al relacionar las experiencias obtenidas en la manipulación de los objetos. ejemplo, el niño diferencia entre un objeto de textura áspera con uno de textura lisa y establece que son diferentes" (pág. 23). Las relaciones lógico - matemáticas es la necesidad de manipular diferentes características que se hayan en su entorno, de una manera interrelacionada de la lógica con las matemáticas y viceversa, no obstante, su aprendizaje resulta compleja por las distintas capacidades que se implementan.

Las relaciones lógico - matemáticas es un proceso complejo que garantiza la formación del niño, por eso Piaget relaciona el desarrollo con el medio de interacción, Silvia, Mendoza (2013) cita a Piaget "La gran labor realizada ha puesto de manifiesto que el pensamiento lógico alcanza su desarrollo por la interacción con el organismo y el sistema materia energía, favorecida por la posesión de determinadas estructuras lingüísticas y por la acción del medio ambiente cultural" (pág. 31).

Las relaciones lógico/matemáticas, que refiere el desarrollo de las fases cognitivas con los que el alumno descubre y entiende su entorno y actúa sobre él para aumentar los diferentes aspectos del pensamiento. Este proceso debe hacer que los niños posean nociones superficiales de tiempo, cantidad, espacio, textura, forma, tamaño y color, a través de la interacción con los diferentes elementos a su alrededor y de experiencias que ayude a la construcción de nociones y relaciones para implementarla en la resolución de problemas y en la búsqueda ardua de nuevos conocimientos. Se encuentra en el eje de desarrollo y aprendizaje del descubrimiento del medio natural y cultural, en el Ámbito de desarrollo y aprendizaje de las Relaciones lógico/matemáticas.

\section{Materiales y métodos}

Se realizó una investigación de campo, descriptiva, explicativa, cuanti-cualitativa y correlacional. Los métodos empleados fueron el empírico, teórico, inductivo. Deductivo, estadístico y profesional; las técnicas e instrumentos utilizados fueron la observación, encuesta y entrevista con el diseño de sus respectivos instrumentos con el fin de determinar: el problema con la ficha de observación, la importancia e incidencia de los juegos de concentración en el desarrollo 
lógico-matemático con los instrumentos de encuesta y entrevista; se realizó una pre y post evaluación con el test pedagógico, en donde se diagnosticó el nivel de desarrollo lógicomatemático de los niños, antes y después de la aplicación de . una guía didáctica a los sujetos de estudio.

El programa fue autofinanciado por los investigadores y contó con el apoyo del director de la escuela y sus docentes, se sustentó en la base legal de la Ley Orgánica de Educación Intercultural y Bilingüe del Ecuador, (LOEI, 2016), que en su artículo 7 indica los derechos de los estudiantes "Recibir formación integral y científica, que contribuya al pleno desarrollo de su personalidad, capacidades y potencialidades, respetando sus derechos, libertades fundamentales y promoviendo la igualdad de género, la no discriminación, la valoración de las diversidades, la participación, autonomía y cooperación”

El programa didáctico se aplicó a niños de 4 a 5 años y tuvo una duración de 4 semanas y una sesión de clase diaria de 30 minutos. El objetivo del programa fue el desarrollo lógicomatemático de los niños de 4 a 5 años de la escuela de Educación Básica Mixta, "Eloy Alfaro", del Cantón Daule el Ecuador en el periodo 2017-2018. Todas las actividades están supeditadas a las necesidades de los niños. La guía se la encuentra en el link: https://es.slideshare.net/leninmendietatoledo/programa-de-juegos-de-concentracin-en-eldesarrollo-de-las-relcaiones-lgicomatemticas/edit?src=slideview\&type=privacy.

\section{Resultados}

\section{Ficha de observación}

Gráfico 1. Proponen juegos y construyen sus propias reglas interactuando con otros.

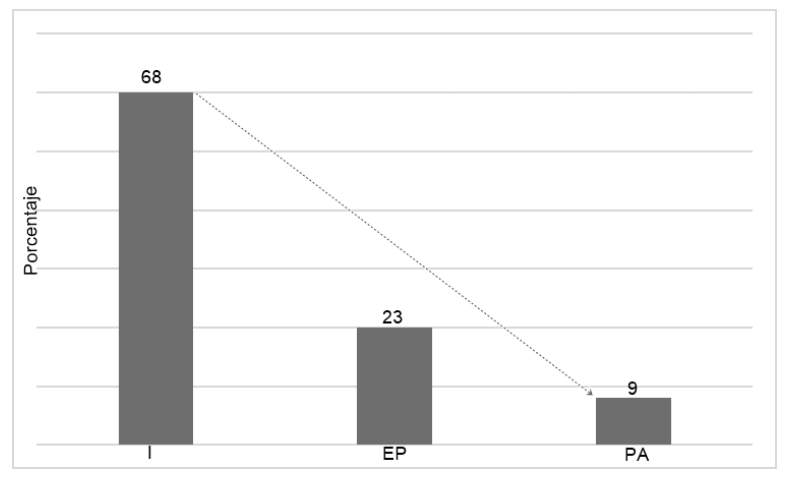

Fuente: Elaboración propia 
Análisis. El 68\% de niños aún está iniciando en proponer juegos y construir sus propias reglas interactuando con otros; el $23 \%$ se encuentra en proceso y el $9 \%$ en progreso adecuado.

Gráfico 2. Clasifican objetos con los atributos (tamaño, color o forma)

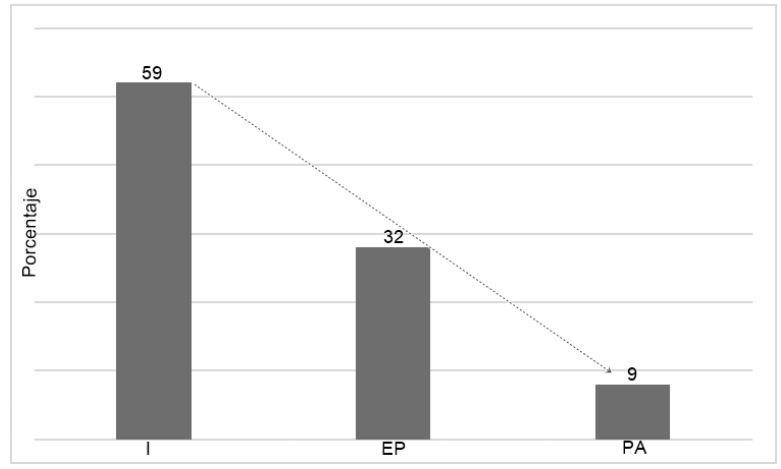

Fuente: Elaboración propia

Análisis. El 59\% aún no clasifican objetos con los atributos (tamaño, color o forma) ya que están iniciando; el $32 \%$ se encuentra en proceso y el $9 \%$ en progreso adecuado

\section{Encuesta}

Gráfico 3. ¿En qué ayudará a mejorar los juegos de concentración el aprendizaje del niño?

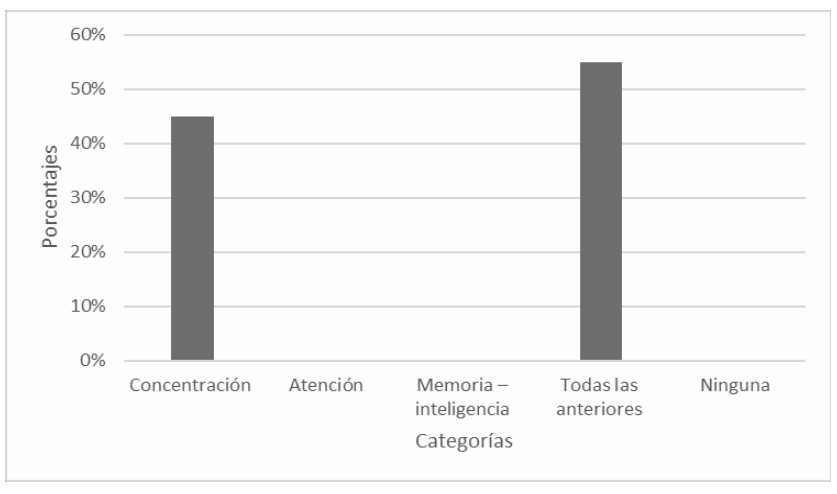

Fuente: Elaboración propia

Análisis: se puede inferir que los docentes consideran que los juegos de concentración ayudarán al aprendizaje de los niños en un $45 \%$ en la concentración y 55\% entre la memoria, atención y concentración. 
Gráfico 4. ¿Es importante que se utilice juegos de concentración en niños de 4 a 5 años para su desarrollo del pensamiento?

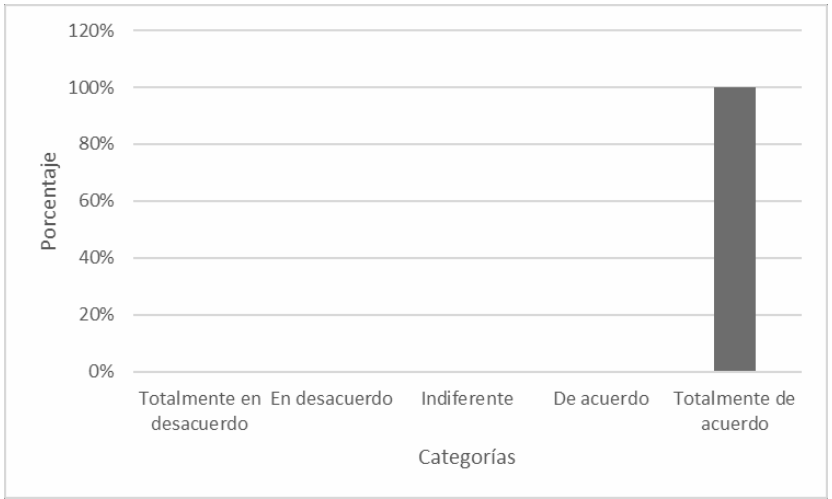

Fuente: Elaboración propia

Análisis. El 100\% de encuestados indicó que es importante que se utilice juegos de concentración en niños de 4 a 5 años para su desarrollo del pensamiento

Gráfico 5. ¿Con que frecuencia el docente se interesa por el desarrollo de los juegos de concentración en los niños de 4 a 5 años?

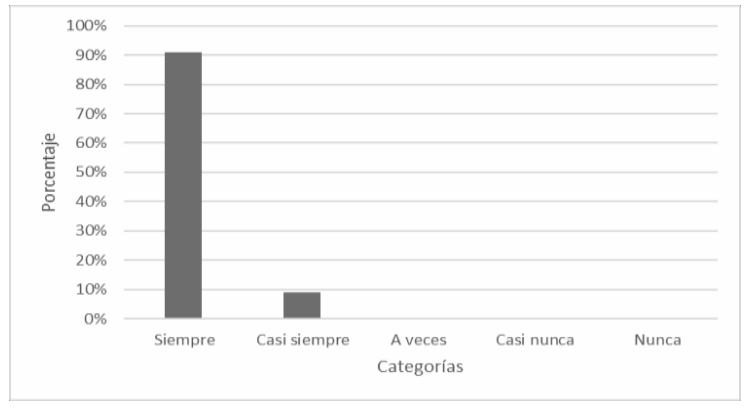

Fuente: Elaboración propia

Análisis. El 91\% de los docentes indicó que siempre se interesa por el desarrollo de los juegos de concentración en los niños de 4 a 5 años; el 19\% indicó que se interesa casi siempre en un 9\% 
Gráfico 6. ¿Qué nociones básicas facilitan las habilidades del pensamiento para la solución de problemas sencillos para el desarrollo de las relaciones lógico - matemáticas?

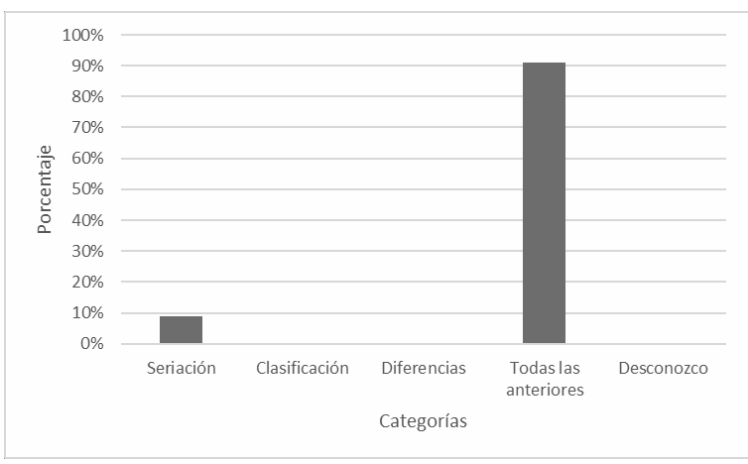

Fuente: Elaboración propia

Análisis. El 100\% de docentes indica que la seriación, clasificación y diferencias son nociones básicas que facilitan las habilidades del pensamiento para el desarrollo de las relaciones lógico matemáticas.

Gráfico 7. ¿El diseño y aplicación de una guía de juegos de concentración ayudará en el desarrollo de las relaciones lógico - matemáticas en los niños de 4 a 5 años?

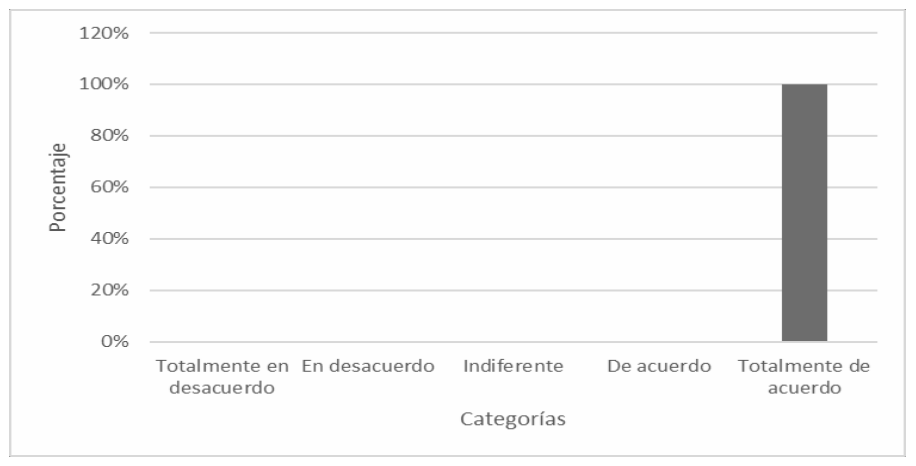

Fuente: Elaboración propia

Análisis. El 100\% de los encuestados está de acuerdo en que los juegos de concentración ayudan al desarrollo de las relaciones lógico-matemático en los niños. 


\section{Entrevista}

Fuente: directora de la escuela

Pregunta (P)

P. ¿Considera usted que es importante el desarrollo de los juegos de concentración?

Respuesta (R). Si es importante porque estimula la participación grupal de los niños para que interactúen entre ellos y puedan desarrollar mejor su concentración para que puedan solucionar problemas sencillos de forma lúdica.

P. ¿En qué cree usted qué ayudará a mejorar los juegos de concentración el aprendizaje del niño?

R. Ayudará en desarrollar su memoria en su concentración y atención donde el niño podrá por medio del jugo de manera práctica para mejorar su capacidad de concentración y desarrollen la capacidad de elaborar decisiones lógicas

P. ¿Cree usted que es necesario el desarrrollo de las relaciones lógico - matemáticas en los niños de 4 a 5 años?

R. Si es importante el desarrollo de las relaciones lógico - matemáticas porque les ayuda a los niños s desarrollar el pensamiento crítico a la hora de realizar problemas

P. ¿Qué nociones básicas facilitan las habilidades del pensamiento para la solución de problemas sencillos para el desarrollo de las relaciones lógico - matemáticas?

R. Las nociones básicas que facilitan las habilidades del pensamiento las encontramos en el currículo donde las ponemos en prácticas por medio de actividades donde estén las seriaciones, clasificaciones, diferencias

P. ¿Cree usted que es importante aplicar una guía didáctica con enfoque metodológico para el desarrollo de las relaciones lógico - matemáticas? 
R. Todo aporte que sea para beneficio de la institución es importante si aportas en una investigación científica que ayudará a los niños de inicial de 4 a 5 años para desarrollo del pensamiento lo es.

Análisis. La entrevista permite interpretar las respuestas como una sólida declaración de la importancia de los juegos de concentración en la estimulación de la parte cognitiva de los niños, así también, en la parte socio afectiva, la misma que se ve fortalecida por la interacción entre pares en juegos que les permite el desarrollo de las relaciones lógico-matemáticas. Coadyuva al desarrollo de la memoria y atención, situación que cada vez es más difícil de lograr, sin embargo, las seriaciones, clasificaciones y diferencias permitirán el logro de los objetivos planteados en la guía didáctica.

\section{Gráfico 8. Test pedagógico}

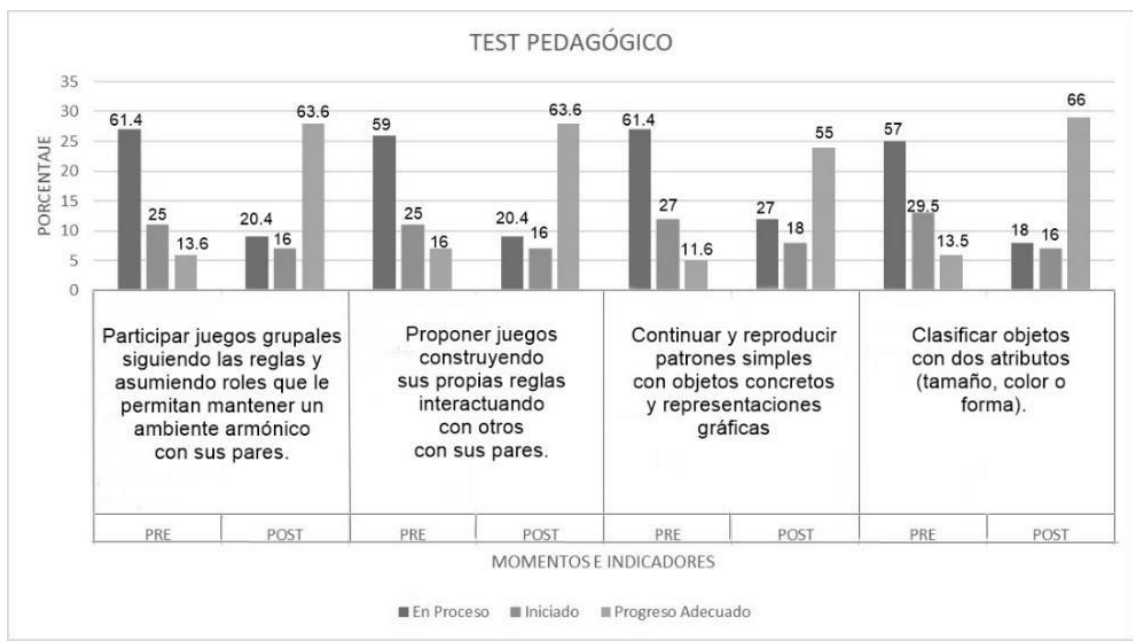

Fuente: Elaboración propia, a partir de la información recogida de los niños de 4 a 5 años de la escuela

Análisis e interpretación. Se evidencia una clara mejoría en los niños en las categorías de: Participar juegos grupales siguiendo las reglas y asumiendo roles que le permitan mantener un ambiente armónico con sus pares, ya que se pasa del 13.6\% al 63.6\%, es decir existe un incremento del 50\% de PA; en Proponer juegos construyendo sus propias reglas interactuando con otros, se incrementa en un $47.6 \%$ en PA; en Continuar y reproducir patrones simples con objetos concretos y representaciones gráficas, se incrementa en un 43,4\%; y en Clasificar objetos 
con dos atributos (tamaño, color o forma), se incrementa en un 52.5\%. Se disminuye los porcentajes de EP e I.

\section{Conclusiones}

Los insumos teóricos permiten tener una visión multidimensional de los beneficios que genera el juego y dentro de ellos los de concentración para el empoderamiento de las relaciones lógicomatemáticas en niños de 4 a 5 años; los resultados de la observación, encuesta y entrevista, permitieron mediante la triangulación científica de evidencias inferir la importancia que tienen los juegos de concentración en el desarrollo de las relaciones lógico-matemáticas, así también, mediante la ficha de observación como instrumento de evaluación del problema, permitió el diseño coherente y congruente de una guía didáctica y su aplicación en veinte sesiones de trabajo con los niños.

Los resultados de la evaluación mediante el test pedagógico en dos momentos pre y post, permitieron determinar las mejoras en el desarrollo de las relaciones lógico-matemáticas en los niños de 4 a 5 años mediante los juegos de concentración.

Se concluye que existe una relación muy estrecha y significativa entre los juegos de concentración en el desarrollo de las relaciones lógico-matemáticas en niños de 4 a 5 años, lo cual permite recomendar que se trabaje con programas o guías didácticas con altos componentes lúdicos en esos grupos etarios.

\section{Referencias Bibliográficas}

Cabanilla Guillén, M. V. (2016). Prácticas de enseñanza lógico - matemáticas de inicial ii en el centro de desarrollo infantil manitas libres. Tesis de titulación, Universidad Politécnica Salesiana, Biblioteca de la Universidad, Quito. Recuperado de: https://dspace.ups.edu.ec/bitstream/123456789/11706/1/UPS-QT09089.pdf. 
Carchi, R. M. (2012). El razonamiento de la lógica y su incidencia en el aprendizaje. Tesis de titulación, Ambato. Recuperado de: http://repositorio.uta.edu.ec/bitstream/123456789/2843/1/tebs_2012_416.pdf.

Delgado Linares, \& , Inmaculada. (2011). El juego infantil y su metodología. Madrid, Madrid, España: paraninfo S.A. Recuperado de: https://books.google.es/books?hl=es\&lr=\&id=sjidLgWM9_8C\&oi=fnd\&pg=P A2\&dq=Definici\%C3\%B3n+de+los+juegos+de+concentraci\%C3\%B3n+\&ots=xFyy0tJH89\&sig $=8 \mathrm{Zuq}_{-}-$

ZXMdbUMbBhhiFD9RPUHE8\#v=onepage\&q=Definici\%C3\%B3n\%20de\%20los\%20juegos $\% 20 \mathrm{de} \% 20$ concentraci\%C3\%B3n\&f=false.

Esteban, J. J. (2012). El juego como estrategia didáctica en expresión plástica. Adaptación a grado infantil, Universidad de Valladolid, España. Recuperado de: https://uvadoc.uva.es/bitstream/10324/1123/1/TFG-B.53.pdf.

Fonseca Ortega, E. (2013). Las actividades lúdicas y su influencia en el desarrollo del pensamiento lógico matemático en los estudiantes del segundo año de educación general básica de la escuela 23 de mayo de la parroquia Chillogallo, Cantón Quito, Provincia de Pichincha. Tesis de titulación, Universidad Técnica de Ambato, Ambato Recuperado de: http://repo.uta.edu.ec/bitstream/123456789/6432/1/FCHE-LEB_1150.pdf.

Giménez, P. V. (2014). Terminología Conceptual para Docentes de Nivel Inicial. Buenos Aires, Buenos Aires, Argentina: DUNKEN. Recuperado de: https://books.google.com.ec/books?id=4BiHBAAAQBAJ\&printsec $=$ frontcover\&dq=terminologia + para+el+docente $\& \mathrm{hl}=\mathrm{es} \&$ sa $=X \& v e d=0$ ahUKEwjAyP7uuPvUAh WB7D4KHcAUDbUQ6AEIJTAA\#v=onepage $\& q=$ terminologia\%20para\%20el\%20docente $\& \mathrm{f}=\mathrm{f}$ alse.

Ibarra Rivas, L. R. (2017). ¿cómo educar? Educarnos. doi: ISNN. 2007-1930

República del Ecuador. Ley Orgánica de Educación Intercultural y Bilingüe del Ecuador (2016) 
Llull, J. (2016). El modelo lúdico en la intervención educativa. El juego infantil y su metodología, 35. Recuperado de: file:///C:/Users/ADMINMINEDUC/Downloads/E1\%20juego\%20infantil_UD01 .pdf.

Prasca medina, M., Pedroza Villarreal, M. V., \& Peña Zuñiga, D. C. (2016). Implementación de actividades lúdico pedagógicas para mejorar la atención y el aprendizaje en los niños y niñas de transición del liceo mixto nuevo siglo soledad - Atlántico - Colombia. Tesis de titulación, Universidad de Cartagena, Biblioteca de la Universidad, Bogotá. Recuperado de: http://190.242.62.234:8080/jspui/bitstream/11227/3031/ 1/proyecto\%20final\%20pedagogia\%20infantil\%20abril-2016.pdf.

Silvia Mendoza, J. E. (2013). Propuesta didáctica para el desarrollo del pensamiento lógico matemático en niños de 5 años. Tesis de titulación, Bogotá. Recuperado de: http://repositorio.pedagogica.edu.co/xmlui/bitstream/handle/123456789/460/TO16309.pdf?seque nce $=1$.

Venegas, García, Venegas Rubiales. (2013). El juego infantil y su metodología. Málaga, Málaga, España: Innovación y Cualificación S.L. Recuperado de: https://books.google.com.ec/books?id=HuCcCIqq1WEC\&prints ec $=$ frontcover $\& \mathrm{dq}=\mathrm{el}+\mathrm{juego}+\mathrm{en}+\mathrm{el}+\mathrm{des}$ arrollo+infantil $\& \mathrm{hl}=\mathrm{es} \& \mathrm{sa}=\mathrm{X} \& \mathrm{ved}=0 \mathrm{ahUKEwjF5MXO}$ yvrUAhVMaD4KHQhTCvMQ6AEIMTAD\#v=twopage\&q\&f=false. 